\title{
Based on Vague Sets of Strawberry Varieties Resistance Comparison
}

\author{
Yan Zhang ${ }^{1}$, Hongxu Wang ${ }^{2}$, and Hongbin Zhang ${ }^{1}$ \\ ${ }^{1}$ College of Biological Science and Technology, \\ Qiongzhou University, \\ Sanya, P.R. China 572022 \\ zy-wzs1991@163.com \\ ${ }^{2}$ Electronic Information Engineering College, \\ Qiongzhou University, \\ Sanya, P.R. China 572022
}

\begin{abstract}
Proposing the similarity measures formula between Vague sets E and G. Concluding Vague resistance analysis. The concrete application steps were: (1) Establishing comprehensive characters set; (2) Screening excellent varieties set; (3) Extracting theory optimal varieties set; (4) The single-value date transformed into Vague date, obtaining different varieties Vague sets; (5)Vague resistance analysis 1,the similarity measures were calculated between the excellent varieties Vague sets and the theory optimal varieties Vague sets, obtaining more suitable for people's needs varieties among excellent varieties; (6) Vague resistance analysis 2, the weighted similarity measures were calculated between the excellent varieties Vague sets and the theory optimal varieties Vague sets, obtaining more suitable for people's needs resistance varieties among excellent varieties. According to specific needs of the problem, selecting and applying the steps (5) or (6). Vague resistance analysis was applied to strawberry varieties resistance analysis, the analysis result was satisfactory. The similarity measures formula between Vague sets E and G was the application basis of Vague resistance analysis.
\end{abstract}

Keywords: Vague sets, Similarity measures formula, Vague resistance analysis, Strawberry varieties, Resistance analysis.

\section{Introduction}

Strawberry is rich nutrition and has a variety of organic acids, minerals and vitamins, especially rich in vitamin c. It is red tender, juicy, sweet delicious and fragrant. It is often known as the empress of the fruit and health food enjoyed by young and old. In recent years, Hainan has actively introduced strawberry fine varieties from home and abroad. This paper intends to use vague resistance analysis to research strawberry varieties resistance analysis, so as to screening fine strawberry varieties more suitable for planting in Hainan. 


\section{Basic Concepts}

\subsection{Vague Sets Definition}

Definition 1[1]. Set non-empty universe Z. For $z \in Z$, regulations interval $\left[t_{E}(z), 1-f_{E}(z)\right]$ for Vague sets $\mathrm{E}$ of $Z$ at dot $z$ Vague value or Vague membership, among them $0 \leq t_{E}(z) \leq 1,0 \leq f_{E}(z) \leq 1$, and meets constraints $t_{E}(z)+f_{E}(z) \leq 1 . t_{E}(z) 、 f_{E}(z) 、 \pi_{E}(z)\left(=1-t_{E}(z)-f_{E}(z)\right)$ is called respectively truth-membership function, false-membership function and uncertain function of Vague set E.

When $Z=\left\{z_{1}, z_{2}, \cdots, z_{n}\right\}$ for discrete universe, its Vague sets $E$ can be written as $E=\sum_{i=1}^{n}\left[t_{E}\left(z_{i}\right), 1-f_{E}\left(z_{i}\right)\right] / z_{i}$, or $E=\sum_{i=1}^{n}\left[t_{e_{i}}, 1-f_{e_{i}}\right] / z_{i}$.

\subsection{Creating Vague Environment}

Creating Vague environment is that original data is transformed into Vague data. It is the application premises of Vague resistance analysis. The following only introduces the definition that the single-value data transformed into theVague data.

Definition 2[2]. Set $Z=\left\{z_{1}, z_{2}, \cdots, z_{n}\right\}$ as discrete universe, $Z$ has sets $E_{i}(i=1,2, \cdots, m), E_{i}$ as the single-value data $z_{i j}(\geq 0)$ that represents the original data of comprehensive characters $z_{j}(j=1,2, \cdots n)$.

a. Vague conditions. $0 \leq t_{i j} \leq 1-f_{i j} \leq 1$;

b. Output conditions. If $0 \leq z_{k j}<z_{i j}$, the single-value data $z_{i j}$ and $z_{k j}$ are respectively transformed into the Vague data $E_{i}\left(z_{j}\right)=z_{i j}=\left[t_{i j}, 1-f_{i j}\right]$ and $E_{k}\left(z_{j}\right)=z_{k j}=\left[t_{k j}, 1-f_{k j}\right]$ meet: $t_{k j} \leq t_{i j}, 1-f_{k j} \leq 1-f_{i j}$. Called single-value data $z_{i j}(\geq 0)$ that meets Vague conditions and output conditions transformed into the Vague data $E_{i}\left(z_{j}\right)=z_{i j}=\left[t_{i j}, 1-f_{i j}\right]$ type of conversion formula for the output-based conversion formula.

c. Investment conditions. If $0 \leq z_{k j}<z_{i j}$, the single-value data $z_{i j}$ and $z_{k j}$ are respectively transformed into the Vague data $E_{i}\left(z_{j}\right)=z_{i j}=\left[t_{i j}, 1-f_{i j}\right]$ and $E_{k}\left(z_{j}\right)=z_{k j}=\left[t_{k j}, 1-f_{k j}\right]$ meet: $t_{k j} \geq t_{i j}, 1-f_{k j} \geq 1-f_{i j}$. Called single-value data $z_{i j}(\geq 0)$ that meets Vague conditions and investment conditions transformed 
into the Vague data $E_{i}\left(z_{j}\right)=z_{i j}=\left[t_{i j}, 1-f_{i j}\right]$ type of conversion formula for the investment-based conversion formula.

Annotation: When the value of comprehensive characters is bigger always better, the output-based conversion formula is suitable used. And when the value of comprehensive characters is smaller always better, the investment-based conversion formula is suitable used.

\subsection{A Kind Vague Membership Data Mining}

Definition 3[3]. The method of a kind Vague membership data mining is: Vague membership $e=\left[t_{e}, 1-f_{e}\right]$, denoted by $t_{e}^{(0)}=t_{e}, f_{e}^{(0)}=f_{e}, \pi_{e}^{(0)}=\pi_{e}=1-t_{e}-f_{e}$. When $m=1,2, \cdots$, and regulations $t_{e}^{(m)}=t_{e} \cdot\left(1+\pi_{e}+\pi_{e}^{2}+\cdots \pi_{e}^{m}\right), f_{e}^{(0)}=f_{e} \cdot\left(1+\pi_{e}+\pi_{e}^{2}+\cdots+\pi_{e}^{m}\right), \pi_{e}^{(m)}=\pi_{e}^{m+1}$.

Lemma 1[3]. $e^{(m)}=\left[t_{e}^{(m)}, 1-f_{e}^{(m)}\right]$ is Vague membership.

Annotation: Definition 3 has put forward the method of Vague membership data mining, it regards Vague membership $e=\left[t_{e}, 1-f_{e}\right]$ mining into Vague membership $e^{(m)}=\left[t_{e}^{(m)}, 1-f_{e}^{(m)}\right] \quad(m=1,2, \cdots)$. This paper by means of Vague membership data mining constructs the new similarity measures between Vague membership.

\subsection{Similarity Measures between Vague Membership}

Definition 4[4]. Set $e=\left[t_{e}, 1-f_{e}\right]$ and $g=\left[t_{g}, 1-f_{g}\right]$ as two Vague membership. The formula $M(e, g)$ is called similarity measures between Vague membership $e$ and $g$. If the formula meets the following conditions:

a. Trivial conditions. $M(e, g) \in[0,1]$;

b. Symmetric conditions. $M(e, g)=M(g, e)$;

c. Reflexive conditions. $M(e, e)=1$;

d. Minimum conditions. When $e=[0,0], g=[1,1]$ or $e=[1,1], g=[0,0]$, they all guarantee $M(e, g)=0$.

The definition of the similarity measures and the weighted similarity measures between Vague sets may be similar to definition 4, here is omitted.

Annotation: $M(e, g)$ expresses similar degree between Vague value $e$ and $g$. Its meaning is that the larger of the value of $M(e, g)$ expresses more similar between Vague value $e$ and $g$; Especially when $M(e, g)$ takes maximum 1, expressing most similar between Vague value $e$ and $g$; The smaller of the value of 
$M(e, g)$ expresses more dissimilarity between Vague value $e$ and $g$; Especially when $M(e, g)$ takes minimum 0 , expressing most dissimilarity between Vague value $e$ and $g$.

\section{New Theorem and New Method}

Theorem 1. $z_{j \min }=\min \left\{z_{1 j}, z_{2 j}, \cdots, z_{m j}\right\}, z_{j \max }=\max \left\{z_{1 j}, z_{2 j}, \cdots, z_{m j}\right\}$. then

$$
\text { a. } E_{i}\left(z_{j}\right)=z_{i j}=\left[t_{i j}, 1-f_{i j}\right]=\left[\frac{z_{i j}-z_{j \min }}{z_{j \max }-z_{j \min }},\left[\frac{z_{i j}-z_{j \min }}{z_{j \max }-z_{j \min }}\right]^{\frac{1}{2}}\right]
$$

It is conversion formula $E_{i}\left(z_{j}\right)=z_{i j}=\left[t_{i j}, 1-f_{i j}\right]$ of single-value data $z_{i j}(\geq 0)$ transformed into Vague data for output-based conversion formula.

b. $E_{i}\left(z_{j}\right)=z_{i j}=\left[t_{i j}, 1-f_{i j}\right]=\left[1-\left[\frac{z_{i j}-z_{j \min }}{z_{j \max }-z_{j \min }}\right]^{\frac{1}{2}}, 1-\frac{z_{i j}-z_{j \min }}{z_{j \max }-z_{j \min }}\right]$

It is conversion formula $E_{i}\left(z_{j}\right)=z_{i j}=\left[t_{i j}, 1-f_{i j}\right]$ of single-value data $z_{i j}(\geq 0)$ transformed into Vague data for investment-based conversion formula.

Theorem 2. Set $e=\left[t_{e}, 1-f_{e}\right]$ and $g=\left[t_{g}, 1-f_{g}\right]$ for two Vague membership. The following formula is similarity measures between Vague membership $e$ and $g(m=0,1,2, \cdots)$ :

$$
M_{m}(e, g)=\frac{1+\min \left\{t_{e}^{(m)}-t_{g}^{(m)}, f_{e}^{(m)}-f_{g}^{(m)}\right\}}{1+\max \left\{t_{e}^{(m)}-t_{g}^{(m)}, f_{g}^{(m)}-f_{g}^{(m)}\right\}} .
$$

Application literature[2] method, not difficult checking out that resolution of formula (3) is higher. Similar theorem 2 obtains the following results.

Theorem 3. Set $Z=\left\{z_{1}, z_{2}, \cdots, z_{n}\right\}$ as the universe, $Z$ has Vague sets $E=\sum_{i=1}^{n}\left[t_{E}\left(z_{i}\right), 1-f_{E}\left(z_{i}\right)\right] / z_{i}$ and $G=\sum_{i=1}^{n}\left[t_{G}\left(z_{i}\right), 1-f_{G}\left(z_{i}\right)\right] / z_{i}$.

Abbrevd. $E=\sum_{i=1}^{n}\left[t_{e_{i}}, 1-f_{e_{i}}\right] / z_{i}, G=\sum_{i=1}^{n}\left[t_{g_{i}}, 1-f_{g_{i}}\right] / z_{i}$.

The following formula is similarity measures between Vague sets $E$ and $G(m=0,1,2, \cdots)$ : 


$$
M_{m}(E, G)=\frac{1}{n} \sum_{i=1}^{n} \frac{1+\min \left\{t_{e_{i}}^{(m)}-t_{g_{i}}^{(m)}, f_{e_{i}}^{(m)}-f_{g_{i}}^{(m)}\right\}}{1+\max \left\{t_{e_{i}}^{(m)}-t_{g_{i}}^{(m)}, f_{e_{i}}^{(m)}-f_{g_{i}}^{(m)}\right\}} .
$$

Theorem 4. Set element $z_{i}$ weight $0 \leq w_{i} \leq 1$, and $\sum_{i=1}^{n} w_{i}=1$. On conditions theorem 3, the following formula is weighted similarity measures between Vague sets $E$ and $G(m=0,1,2, \cdots)$ :

$$
W M_{m}(E, G)=\sum_{i=1}^{n} w_{i} \cdot \frac{1+\min \left\{t_{e_{i}}^{(m)}-t_{g_{i}}^{(m)}, f_{e_{i}}^{(m)}-f_{g_{i}}^{(m)}\right\}}{1+\max \left\{t_{e_{i}}^{(m)}-t_{g_{i}}^{(m)}, f_{e_{i}}^{(m)}-f_{g_{i}}^{(m)}\right\}} .
$$

\section{Vague Resistance Analysis}

Concluding literature[4] Vague sets comprehensive decision rules for Vague resistance analysis. The concrete application steps are: (1)Establishing comprehensive characters set; (2) Screening excellent varieties set; (3) Extracting theory optimal varieties set; (4) The single-value date transformed into Vague date, obtaining different varieties Vague sets; (5)Vague resistance analysis 1,the similarity measures are calculated between the excellent varieties Vague sets and the theory optimal varieties Vague sets, obtaining more suitable for people's needs varieties among excellent varieties; (6)Vague resistance analysis 2, the weighted similarity measures are calculated between the excellent varieties Vague sets and the theory optimal varieties Vague sets, obtaining more suitable for people's needs resistance varieties among excellent varieties. According to specific needs of the problem, selecting and applying the steps (5) or (6).

\section{Strawberry Varieties Resistance Comparison}

Strawberry cultivation range is very wide, in recent years, Hainan has actively introduced strawberry fine varieties from home and abroad. Selection excellent comprehensive characters strawberry varieties is main work for improving strawberry cultivation yield, therefore, we screened five varieties for Frandy, Maiterli, Kinuama, Rafi, Fengxiang and developed five varieties resistance comparison experiment. We applied Vague resistance analysis to analyse, in order to select excellent varieties more appropriate Hainan plastic greenhouse cultivation, to improve strawberry cultivated benefit and economic benefit.

\subsection{Establishing Comprehensive Characters Set}

Establishing comprehensive characters set $Z=\left\{z_{1}, z_{2}, \cdots, z_{8}\right\}$ :

Index $z_{1}$ : survival rate of seedlings $(\%) ; z_{2}$ : survival rate of plant $(\%) ; z_{3}$ : yield $\left(t / \mathrm{hm}^{2}\right) ; z_{4}$ : fruit commodity rate(\%); $z_{5}$ : leaves heating-damage rate(\%); 
$z_{6}$ : young fruit freezing-damage rate $(\%) ; z_{7}:$ blight incidence $(\%) ; z_{8}:$ grey cinerea incidence $(\%)$.

\subsection{Screening Excellent Varieties Set}

Selecting Hainan plastic greenhouse cultivation main fine varieties for excellent varieties sets $E=\left\{E_{1}, E_{2}, E_{3}, E_{4}, E_{5}\right\}$, among them $E_{1}$ : Frandy; $E_{2}$ : Fengxiang; $E_{3}$ : Maiterli; $E_{4}$ : Kinuama; $E_{5}$ : Rafi. They all are sets of comprehensive characters set $Z=\left\{z_{1}, z_{2}, \cdots, z_{8}\right\}$. Resistance comparison experiment original data shown in Table 1.

\subsection{Extracting Theory Optimal Varieties Set}

Because the value of $z_{1}, z_{2}, z_{3}, z_{4}$ is bigger always better, the value of $z_{5}, z_{6}, z_{7}, z_{8}$ is smaller always better. So extraction theory optimal varieties $G$ concrete data shown in Table 1 .

Table 1. The original data of resistance comparison experiment

\begin{tabular}{ccccccc}
\hline & $E_{1}$ & $E_{2}$ & $E_{3}$ & $E_{4}$ & $E_{5}$ & $G$ \\
\hline$z_{1}$ & 96.60 & 92.20 & 97.30 & 95.30 & 94.80 & 97.30 \\
$z_{2}$ & 93.80 & 90.70 & 96.60 & 93.80 & 91.10 & 96.60 \\
$z_{3}$ & 15.79 & 11.38 & 16.00 & 11.94 & 13.91 & 16.00 \\
$z_{4}$ & 87.80 & 71.40 & 81.20 & 72.20 & 86.40 & 87.80 \\
$z_{5}$ & 9.20 & 28.10 & 6.60 & 24.50 & 19.70 & 6.60 \\
$z_{6}$ & 52.10 & 60.80 & 54.80 & 49.40 & 55.50 & 49.40 \\
$z_{7}$ & 16.60 & 8.50 & 5.80 & 5.80 & 27.20 & 5.80 \\
$z_{8}$ & 8.00 & 18.80 & 11.60 & 15.20 & 6.90 & 6.90 \\
\hline
\end{tabular}

\subsection{The Single-Value Date Transformed into Vague Date, Obtaining Different Varieties Vague Sets}

Can be seen from 4.3, application formula(1) to comprehensive characters $z_{1}$, $z_{2}, z_{3}, z_{4}$, application formula(2) to comprehensive characters $z_{5}, z_{6}, z_{7}, z_{8}$, they make the original data in Table 1 transform into Vague data and obtain different varieties Vague sets(see table 2). 


\subsection{Vague Resistance Analysis 1}

Application formula(4)(take $m=2$ ) calculates the similarity measures between the excellent varieties Vague sets and the theory optimal varieties Vague sets, the results show:

$M_{2}\left(E_{1}, G\right)=0.70, M_{2}\left(E_{2}, G\right)=0.09, M_{2}\left(E_{3}, G\right)=0.77$, $M_{2}\left(E_{4}, G\right)=0.42, M_{2}\left(E_{5}, G\right)=0.41$. The preferential order of strawberry varieties adaptation Hainan is: $\operatorname{Maiterli}\left(E_{3}\right), \operatorname{Frandy}\left(E_{1}\right), \operatorname{Kinuama}\left(E_{4}\right), \operatorname{Rafi}\left(E_{5}\right)$, Fengxiang $\left(E_{2}\right)$. First choice Maiterli.

\subsection{Vague Resistance Analysis 2}

The cold resistant strawberry varieties need to be considered at slightly cold incidental heavy fetch in middle of Hainan.

Application formula(5) (take $m=2$ ), take weight:

$w_{1}=0.1, w_{2}=0.1, w_{3}=0.1, w_{4}=0.1, w_{5}=0.1, w_{6}=0.3, w_{7}=0.1, w_{8}=0.1$ the weighted similarity measures are calculated between the excellent varieties Vague sets and the theory optimal varieties Vague sets, the results show:

$W M_{2}\left(E_{1}, G\right)=0.67, W M_{2}\left(E_{2}, G\right)=0.07, W M_{2}\left(E_{3}, G\right)=0.66, W M_{2}\left(E_{4}, G\right)=0.53$, $W M_{2}\left(E_{5}, G\right)=0.37$. The preferential order of strawberry varieties adaptation slightly cold incidental heavy fetch in middle of Hainan is: Frandy( $\left.E_{1}\right)$, Maiterli( $\left.E_{3}\right)$, Kinuama $\left(E_{4}\right), \operatorname{Rafi}\left(E_{5}\right)$, Fengxiang $\left(E_{2}\right)$. First choice Frandy.

Table 2. Vague data of resistance comparison experiment

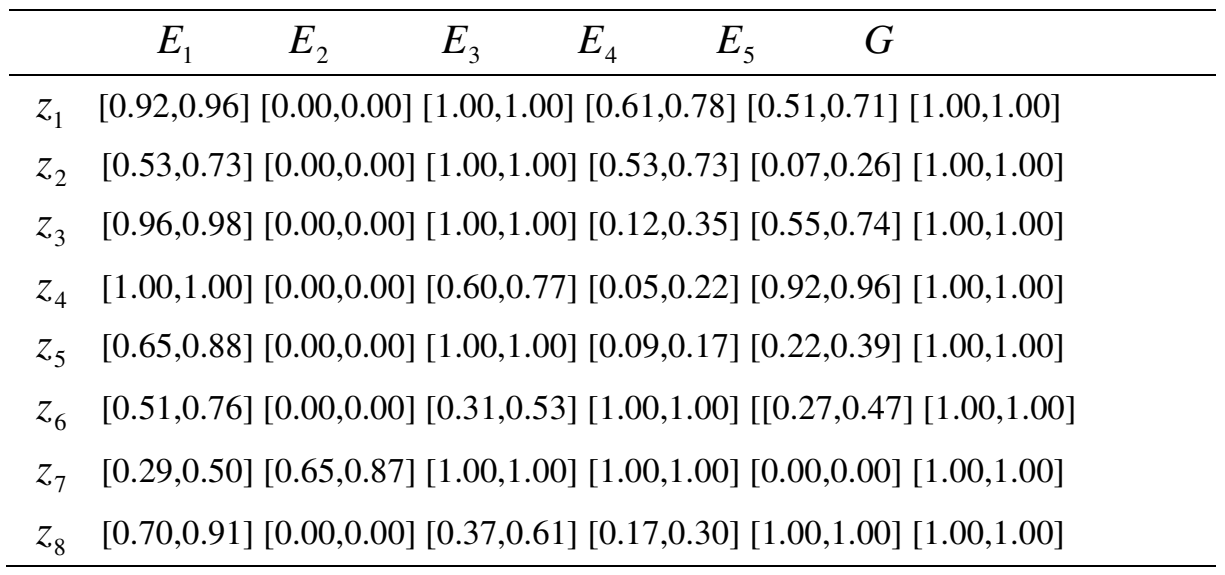




\section{Conclusion}

Through strawberry varieties Vague resistance analysis, it gave a new method to study such problems, but also it enriched Vague pattern recognition theory. The method was a kind of pattern recognition method, it made pattern recognition between excellent varieties and theory optimal varieties. The recognition tool was the similarity measures formula between Vague sets and the weighted similarity measures formula between Vague sets. New formula(1) $(5)$ was used in Vague resistance analysis, especially new formula(4) $\sim(5)$ was corroborated, such formula was indispensable for Vague pattern recognition theory[5].

Fund project: Hainan Natural Science Fundation of China under Grant No.610224; Hainan Social Development Technology Special Fundation of China under Grant No.2010SF004.

\section{References}

[1] Gau, W., Buehrer, D.J.: Vague Sets. IEEE Transactions on Systems. Man and Cybernetics 23(2), 610-614 (1993)

[2] Wang, H.: Comprehensive evaluation of new wheat varieties applying Vague optimized decision-making method. Computer Engineering and Applications 47(12), 210-212 (2011)

[3] Liu, H., Wang, F.: Transformations and Similarity Measures of Vague Sets. Computer Engineering and Applications 40(32), 79-81, 84 (2004)

[4] Wang, H.: Synthesis decision rule of vague sets and its application in scheme optimum seeking. Computer Engineering and Applications 46(27), 145-147 (2010)

[5] Liu, H.: Basis of fuzzy pattern recognition-similarity measures. Pattern Recognition and Artificial Intelligence 17(2), 141-145 (2004) 